\title{
Study of Hyperon and Antihyperon Production in Deep Inelastic Muon Scattering
}

\author{
Natalia ROSSIYSKAYA*, on behalf of the COMPASS collaboration \\ LHEP JINR \\ E-mail: Natalia.Rossiyskaya@cern.ch
}

The yields of heavy hyperons and antihyperons have been studied in deep inelastic scattering (DIS) at the COMPASS experiment at CERN. The relative yields of $\Sigma(1385)^{+}, \Sigma(1385)^{-}$, $\bar{\Sigma}(1385)^{-}, \bar{\Sigma}(1385)^{+}, \Xi(1321)^{-}$, and $\bar{\Xi}(1321)^{+}$hyperons decaying into $\Lambda(\bar{\Lambda}) \pi$ were measured. The ratios of $\Sigma^{ \pm} / \Lambda, \bar{\Sigma}^{ \pm} / \bar{\Lambda}, \Xi^{-} / \Lambda$ and $\bar{\Xi}^{+} / \bar{\Lambda}$ were determined. All yield ratios were found to be in the range $3.8 \%$ to $5.6 \%$. Within the relative uncertainties of about $10 \%$, the yield ratios for hyperons and antihyperons are quite similar. The measured yields were used to tune the parameters of the LEPTO generator simulating the DIS events.

The European Physical Society Conference on High Energy Physics

18-24 July, 2013

Stockholm, Sweden

\footnotetext{
* Speaker.
} 


\section{Experimental hyperons samples}

The study of hyperon production in deep inelastic scattering is important for a better understanding of the role of strange quarks in the nucleon structure and in the hadronization process. The lightest hyperon, the $\Lambda$ baryon, was studied in most detail. In addition to $\Lambda$ from direct production, a significant fraction of $\Lambda$ particles originates from the decay of heavier hyperons such as $\Sigma^{0}, \Sigma^{*}$, or $\Xi$. Information on the production of these heavy hyperons with muon or electron beams is still missing. To our knowledge, the production of the antiparticles, $\bar{\Sigma}^{*-}, \bar{\Sigma}^{*+}$, and $\bar{\Xi}$ has never been studied in DIS.

The data used in the present analysis were collected by the COMPASS Collaboration [1] at CERN during the years 2003-2004. The experiment was performed at the CERN M2 muon beam line. The average beam momentum was $160 \mathrm{GeV} / c$. DIS events are selected by cuts on the fourmomentum squared of the virtual photon, $Q^{2}>1(\mathrm{GeV} / \mathrm{c})^{2}$, and on the fractional energy $y$ of the virtual photon, $0.2<y<0.9$. The resulting data sample consists of $3.12 \cdot 10^{8}$ events. The $\Lambda$ and $\bar{\Lambda}$ hyperons are identified by their decays into $p \pi^{-}$and $\bar{p} \pi^{+}$, respectively. The total numbers of reconstructed $\Lambda$ and $\bar{\Lambda}$ hyperons [2] are $N(\Lambda)=112449 \pm 418$ and $N(\bar{\Lambda})=66685 \pm 350$. They represent an improvement of an order of magnitude with respect to previous experiments [3]. The analysis was done in the current fragmentation region $x_{F}>0.05$.

The $\Lambda$ hyperons in the resulting event samples are either directly produced or originate from the decay of heavier hyperons. The branching ratios of $\Sigma^{*}, \Xi$ hyperons and their antiparticles which decay to $\Lambda(\bar{\Lambda}) \pi^{ \pm}$are equal to $87.5 \%$ or $99.9 \%$, respectively. Since the $\Sigma^{*}$ decays via strong interaction, the production and decay vertices are indistinguishable. In contrast, the $\Xi$ hyperon decays via weak interaction, such that the decay vertex is clearly separated from the production vertex.

In order to search for $\Sigma^{*}$ and $\Xi$ the $p \pi^{-}\left(\bar{p} \pi^{+}\right)$pairs within a $\pm 2 \sigma$ mass interval from the mean value of the $\Lambda(\bar{\Lambda})$ peak were then combined with a charged track from the primary vertex (case of $\Sigma^{*}$ ) or charged track not attached to the primary vertex (case of $\Xi$ ). The number of $\Sigma^{*}$ found to be: $N\left(\Sigma^{*+}\right)=3631 \pm 333, N\left(\Sigma^{*-}\right)=2970 \pm 490, N\left(\bar{\Sigma}^{*-}\right)=2173 \pm 222$ and $N\left(\bar{\Sigma}^{*+}\right)=1889 \pm 265$. The reconstructed numbers of $\Xi$ hyperons are: $N\left(\Xi^{-}\right)=2320 \pm 68$ and $N\left(\bar{\Xi}^{+}\right)=1147 \pm 49$.

In order to extract the yield ratios of heavy hyperons to $\Lambda$ baryons, the ratios of the corresponding acceptances had to be evaluated. The resulting values of the acceptance ratios for $\Sigma^{*}$ to $\Lambda$ and for $\Xi$ to $\Lambda$ are 0.67 and 0.42 , respectively.

\section{Discussion of results}

The ratios of the acceptance-corrected yields of $\Sigma^{*}$ and $\Xi$ to that of $\Lambda$ hyperons are given in Table 1 along with their statistical and systematic uncertainties.

The ratios of production yields of hyperons and antihyperons to those of $\Lambda$ and $\bar{\Lambda}$ are obtained for the first time in charged lepton DIS reactions. Earlier, only hyperon to $\Lambda$ yields, but no yields for antiparticles, were measured in neutrino DIS by the NOMAD Collaboration [5]. The NOMAD values are also shown in Table 1. We note that COMPASS has collected considerably larger (from 30 to 130 times) samples of hyperons than NOMAD in the current fragmentation region. It is interesting to compare the charged lepton and the neutrino data despite the different underlying 
interactions. COMPASS measures similar values for the $\Sigma^{*+} / \Lambda$ and $\Sigma^{*-} / \Lambda$ ratios. Taking into account experimental uncertainties, the same conclusion is valid for NOMAD data but with NOMAD values being a factor of two smaller than the COMPASS ones. Finally, within uncertainties, the $\Xi^{-} / \Lambda$ yield ratio measured by NOMAD is consistent with zero, while COMPASS gives comparable and non-zero values for $\Xi^{-} / \Lambda$ and $\bar{\Xi}^{+} / \bar{\Lambda}$ ratios. The large experimental uncertainties in the NOMAD measurements prevent us from drawing conclusions about heavy hyperon production in charged lepton DIS as compared to neutrino DIS.

Table 1: The heavy hyperon to $\Lambda$ and antihyperon to $\bar{\Lambda}$ yield ratios in lepton DIS. The results are compared with those from NOMAD [5] neutrino DIS data in the current fragmentation region. The average neutrino energy of charged current interactions was $45.3 \mathrm{GeV}$.

\begin{tabular}{lcc}
\hline Ratios & This work & NOMAD \\
\hline$\Sigma^{*+} / \Lambda$ & $0.055 \pm 0.005($ stat $) \pm 0.005($ syst $)$ & $0.025 \pm 0.019$ \\
$\bar{\Sigma}^{*-} / \bar{\Lambda}$ & $0.047 \pm 0.006($ stat $) \pm 0.006($ syst $)$ & - \\
$\Sigma^{*-} / \Lambda$ & $0.056 \pm 0.009($ stat $) \pm 0.007($ syst $)$ & $0.037 \pm 0.015$ \\
$\bar{\Sigma}^{*+} / \bar{\Lambda}$ & $0.039 \pm 0.006($ stat $) \pm 0.006($ syst $)$ & - \\
$\Xi^{-} / \Lambda$ & $0.038 \pm 0.003($ stat $) \pm 0.002($ syst $)$ & $0.007 \pm 0.007$ \\
$\bar{\Xi}^{+} / \bar{\Lambda}$ & $0.043 \pm 0.004($ stat $) \pm 0.002($ syst $)$ & - \\
\hline
\end{tabular}

In order to reproduce better the measured ratios, the LEPTO/ JETSET 7.4 parameters PARJ(1) - PARJ(5), PARJ(7) [6] related to the production yields of strange baryons were tuned. A study of the MC distributions of the common SIDIS $Q^{2}$ and $W$ and the baryon variables $z$ and $p_{T}$ for $\Lambda, \Sigma^{*}$, $\Xi$ and their antiparticles was performed. The distributions of two MC data sets, with default and tuned parameters, were found to be consistent within errors. The $Q^{2}, W, z$ and $p_{T}$ ratios of real data and Monte Carlo samples with both tuned and default parameters are similar without strong deviations from unity.

The heavy hyperon to $\Lambda$ and heavy antihyperon to $\bar{\Lambda}$ yield ratios were measured for the first time in charged lepton deep-inelastic scattering. The obtained results imply that some parameters of the LEPTO code, which are associated with strange quark production and fragmentation in charged lepton DIS processes, should be substantially modified. Using the tuned LEPTO parameters, the fractions of indirectly produced $\Lambda$ and $\bar{\Lambda}$ hyperons were found to be $37 \%$ and $32 \%$, respectively.

\section{References}

[1] P. Abbon et al. (COMPASS Collaboration), Nucl. Instrum. Meth. A577 455 (2007)

[2] P. Abbon et al. (COMPASS Collaboration), CERN-PH-EP-2013-052, arXiv:1304.0952

[3] A. Airapetian et al. (HERMES Collaboration), Phys. Rev. D74 072004 (2006); P. Astier et al. (NOMAD Collaboration), Nucl. Phys. B605 3 (2001); M.R. Adams et al. (E665 Collaboration), Eur. Phys. J. C17 263 (2000); Quinhua Xu (STAR Collaboration), SPIN06, 915 (2006) 428, hep-ex/0612035

[4] A.E.G. Ingelman, A. Edin, J. Rathsman, Comp. Phys. Commun. 101108 (1997)

[5] P. Astier et al. (NOMAD Collaboration), Nucl.Phys. B621 3 (2002)

[6] T. Sjöstrand, Comp. Phys. Commun. 8274 (1994) 